\title{
A Five-Gene Prognostic Nomogram Predicting Disease-Free Survival of Differentiated Thyroid Cancer
}

\author{
Pan Ruchong $\mathbb{D}^{1},{ }^{1}$ Tang Haiping $\mathbb{D}^{2},{ }^{2}$ and Wang Xiang $\mathbb{D}^{2}$ \\ ${ }^{1}$ Faculty of Medical Technology, Chongqing Three Gorges Medical College, Chongqing, China \\ ${ }^{2}$ Transfusion Section, Chongqing University Three Gorges Hospital, Chongqing, China \\ Correspondence should be addressed to Wang Xiang; 13798849057@163.com
}

Received 10 January 2021; Accepted 27 May 2021; Published 16 June 2021

Academic Editor: Chiara Nicolazzo

Copyright (C) 2021 Pan Ruchong et al. This is an open access article distributed under the Creative Commons Attribution License, which permits unrestricted use, distribution, and reproduction in any medium, provided the original work is properly cited.

\begin{abstract}
Background. Differentiated thyroid cancer (DTC) is the most common type of thyroid tumor with a high recurrence rate. Here, we developed a nomogram to effectively predict postoperative disease-free survival (DFS) in DTC patients. Methods. The mRNA expressions and clinical data of DTC patients were downloaded from the Cancer Genome Atlas (TCGA) and the Gene Expression Omnibus (GEO) database. Seventy percent of patients were randomly selected as the training dataset, and thirty percent of patients were classified into the testing dataset. Multivariate Cox regression analysis was adopted to establish a nomogram to predict 1-year, 3-year, and 5-year DFS rate of DTC patients. Results. A five-gene signature comprised of TENM1, FN1, APOD, F12, and BTNL8 genes was established to predict the DFS rate of DTC patients. Results from the concordance index (C-index), area under curve (AUC), and calibration curve showed that both the training dataset and the testing dataset exhibited good prediction ability, and they were superior to other traditional models. The risk score and distant metastasis (M) of the five-gene signature were independent risk factors that affected DTC recurrence. A nomogram that could predict 1-year, 3year, and 5-year DFS rate of DTC patients was established with a C-index of 0.801 (95\% CI: 0.736, 0.866). Conclusion. Our study developed a prediction model based on the gene expression and clinical characteristics to predict the DFS rate of DTC patients, which may be applied to more accurately assess patient prognosis and individualized treatment.
\end{abstract}

\section{Introduction}

Differentiated thyroid cancer (DTC) is the most common tumor in the head and neck area and accounts for approximately $90 \%$ of all cases. DTC is composed of papillary thyroid carcinoma (PTC) and follicular thyroid carcinoma (FTC), which both originate from follicular cells of the thyroid $[1,2]$. Although the 10 -year mortality rate of DTC is only $1.7 \%$, the postoperative recurrence rate is up to $35 \%$, and the mortality rate of recurrent patients is $48 \%$ [3-5]. The ability to precisely predict individual risk of recurrence has become an important and effective measure to prevent the recurrence of DTC. Currently, the commonly used risk stratification includes the American Joint Committee on Cancer (AJCC) staging system冈the American Thyroid Association (ATA) staging system, and the European Thyroid Association (ETA) staging system [6-8]. Although these traditional risk stratification systems are useful for predicting overall patient outcome, it is difficult to apply them for individualized and accurate prediction. Due to the emergence of molecular tumor profiling, analysis of prognostic-related genes has significantly progressed, making it possible to accurately predict the prognosis of patients at the molecular level. In previous studies of cancer prediction analysis, the prediction system integrated gene signature and clinical characteristics, which provides more accurate and reliable prediction results in contrast to the traditional staging systems [9-11].

At present, nomogram has been accepted as prognostic evaluation method based on evidence-based and precision medicine, and it has been widely used in prognosis analysis of various tumors [12-14]. In this study, we analyzed the mRNA expression of cancer and paracancerous tissue of DTC patients from the Cancer Genome Atlas (TCGA) and Gene Expression Omnibus (GEO) databases to screen for differentially expressed genes (DEGs) related to patient disease-free survival (DFS). Multivariate Cox regression 
analysis was employed to establish a gene signature, which was compared with the traditional and other risk stratification models. Gene signature indicating high- and low-risk groups underwent Kaplan-Meier survival correlation curve analysis and GSEA pathway enrichment analysis. Finally, a nomogram based on gene signature and clinical characteristics was plotted to predict the 1-, 3-, and 5-year DFS rate of patients with DTC.

\section{Materials and Methods}

2.1. Data Source and Screening of Differentially Expressed Genes. The mRNA expression data (HTSeq counts) of cancerous and paracancerous tissue were collected up to March 29, 2020 from the TCGA-THCA (https://cancergenome.nih .gov/), GSE27155, and GSE53157 datasets (CEL files). To obtain the mRNA expression from normal tissue and tumors, data from the GEO database (https://www.ncbi.nlm.nih.gov/ geo/) was downloaded. The edge $R$ package (version3.26.3) and limma package (version 3.38.3) in $R$ (version 3.6.0) were run to eliminate very low-expression genes. The mRNA expression data obtained from TCGA and GEO was normalized to eliminate errors. Then, log (fold change) $>1$ and false discovery rate $($ FDR $)<0.05$ were set to screen DEGs in the cancer and control group. Venn diagrams were employed to determine the intersection between the three datasets to obtain DEGs in common. Gene Ontology (GO) and Kyoto Encyclopedia of Genes and Genomes (KEGG) enrichment of DEGs was conducted by using the DAVID bioinformatic resources (version 6.8; https://david.abcc.ncifcrf.gov/) with $P<0.05$ as the selection condition. The clinical data of patients included DFS time, recurrence status, cancer type, gender, age, tumor AJCC staging, tumor T staging, tumor $\mathrm{N}$ staging, and tumor $\mathrm{M}$ staging [15]. All clinical parameters were based on the AJCC staging system (8th edition) for thyroid cancer, [16] and clinical data with missing information or duplicate samples were excluded.

\subsection{Establishment and Validation Analysis of Gene Signature.} Samples with the normalized mRNA expression data and integrated clinical data were included in the overall analysis. A total of $70 \%$ of the total number of samples were randomly selected as the training dataset [17], and the remaining 30\% of samples served as the testing dataset [18] using the caret package (version 6.0-84). Data from the training dataset were analyzed by univariate Cox regression through the survival package (version 2.44-1.1), and the DEGs related to DFS were screened out using $P<0.05$. In addition, the glmnet package (version 2.0-18) was adopted to perform Lasso regression analysis. Data was randomly simulated 1,000-fold, then crossvalidated to select DEGs with the best predictive ability. The screened DEGs were analyzed by stepwise multivariate Cox regression analysis through the survival package in $R$. Risk score and C-index of the training dataset were calculated, and a gene signature was constructed by combining the regression coefficient and mRNA expression. The gene signature was applied to the TCGA, GSE27155, and GSE53157 datasets to evaluate its ability of distinguishing between cancer and normal tissue by AUC. The DEGs of the gene signature were used for the analysis of pairing differences of the gene expression in cancerous versus paracancerous tissue. The 1-year, 3-year, and 5-year receiver operating characteristic (ROC) curves of patients in the training dataset were plotted via the risk ROC package (version 1.0.3). Then, the gene signature model formula of the training dataset was incorporated into the data of the testing and entire dataset to achieve the ROC curve and AUC values of the data model, respectively. Subsequently, the AUC value was used as a measure to compare the performance of our model with that of other models including the five-gene signature model of Wu et al. [11], AJCC stage model, ATA model, and ETA model. The comparison between the five-gene signature model of $\mathrm{Wu}$ et al. and our model was performed using TCGA-THCA HTSeq count dataset.

2.3. Risk Grouping Analysis of Gene Signature. In the training dataset, the best cutoff point for the risk score was identified using the $X$-tile software (version 3.6.1) in order to divide the data into high-risk and low-risk groups [19]. Kaplan-Meier survival curves of high- and low-risk groups were created and plotted using the $R$ survival package. In addition, a risk curve, a risk scatter chart, and a risk heat map were created to visualize the data of high- and low- risk groups. Then, the best cutoff point of the risk score in the training dataset was validated using the data of the testing and entire dataset. The Kaplan-Meier survival curve was plotted to verify the discriminative performance of the cutoff point. The highand low- risk groups in the entire dataset were included in the gene set enrichment analysis (GSEA) (version 4.0.2 for windows), and the gene annotation set c2.cp.kegg.v7.0.symbols.gmt served as a reference. To obtain the differences in biological function and pathways between high- and lowrisk groups, the results were filtered and analyzed at $P<$ 0.05 and $\mathrm{FDR}<0.25[20]$.

2.4. Establishment and Evaluation Analysis of Predictive Nomogram. Clinical data and risk score values of patients in the entire dataset were extracted and included in the subsequent analysis. A univariate and multivariate Cox regression analysis using the $R$ survival package was employed to screen for independent risk factors that affected the recurrence of DTC patients. Using the rms package (version 5.13.1 ), a nomogram was plotted, which contained all independent risk factors and related clinical data. In addition, a calibration curve was established to evaluate the predictive ability of the nomogram.

2.5. Statistical Analysis. Statistical analysis was performed based on $R$ (version 3.6.0), where categorical variables were analyzed by $\chi^{2}$ test or Fisher's exact test. Continuous variables were analyzed by $t$-test, and multiple groups of continuous variables were analyzed by one-way ANOVA with Bonferroni posthoc test. The difference in prognosis between high- and low-risk groups was analyzed by the log-rank test. Of each relevant factor, the hazard ratio (HR) and 95\% confidence interval (CI) were calculated. Unless otherwise specified, $P<0.05$ was considered statistically significant. 


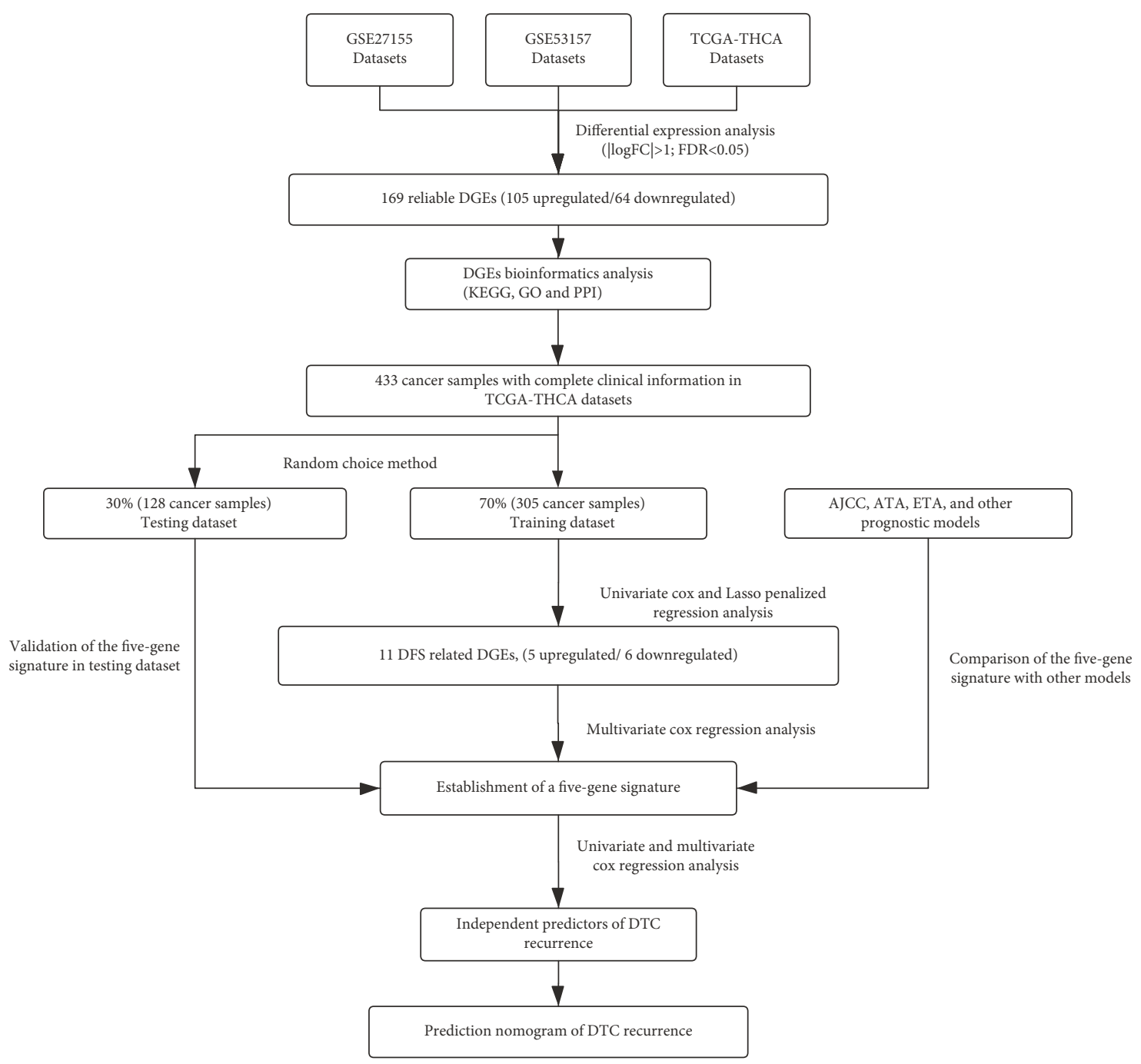

FIGURE 1: Flowchart of establishing the gene signature and prognostic nomogram of DTC patients.

TABLE 1: Details of the datasets used for screening differentially expressed genes.

\begin{tabular}{lcc}
\hline Datasets & Platform & Sample size (tumor/control) \\
\hline GSE27155 & Affymetrix Human Genome U133A Array & Tumor:64/control:4 \\
GSE53157 & Affymetrix Human Genome U133 Plus 2.0 Array & Tumor:11/control:3 \\
TCGA-THCA & Illumina RNA Sequencing & Tumor:509/control:58 \\
\hline
\end{tabular}

\section{Results}

3.1. Data Source and DEG Screening Analysis. The main flow of this study is presented in Figure 1. Table 1 shows the characteristics of the three datasets containing the accession numbers of datasets, sample size, and platforms. When analyzing DEGs of the three datasets in common, 169 DEGs were obtained, including 105 upregulated genes and 64 downregulated genes (Figures 2(a) and 2(b)). GO analysis demonstrated that changes in biological processes of DEGs were mainly enriched in interactions between extracellular matrix (ECM), angiogenesis, BMP signaling pathway, transforming growth factor-beta receptor signaling pathway, and the regulation of the MAPK cascade (Figure 2(c)). Furthermore, enrichment analysis on the KEGG pathway showed that the DEGs were mainly concentrated in cancer pathways and the P53 signaling pathway (Figure 2(d)). Among the relevant clinical data of DTC patients, a total of 433 samples with complete clinical information were selected for analysis.

\subsection{Establishment and Validation of the Five-Gene Signature.} The entire dataset of the TCGA dataset was randomly divided into 305 cases in the training dataset and 128 cases in the testing dataset. Table 2 shows the clinical baseline features of DTC patients in the training and testing datasets. In the training dataset, based on the univariate Cox analysis of 


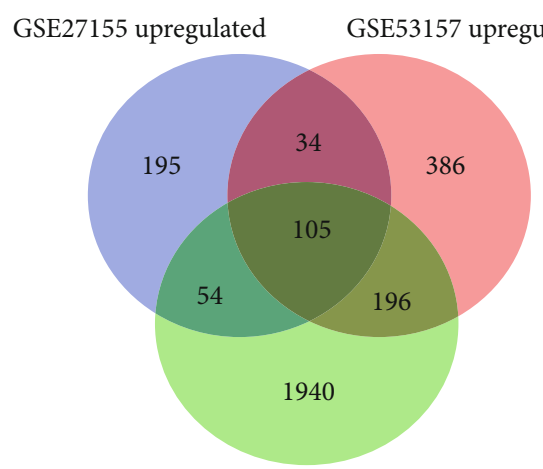

TCGA-THCA upregulated

(a)
GSE27155 downregulated GSE53157 downregulated

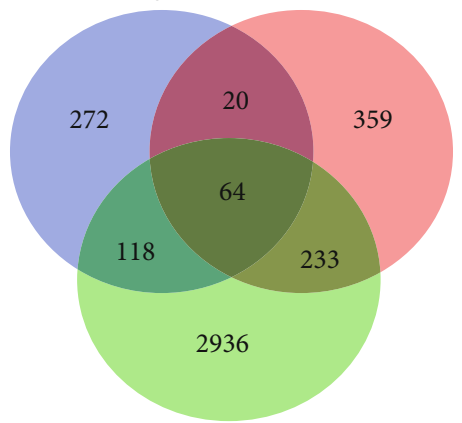

TCGA-THCA downregulated

(b)

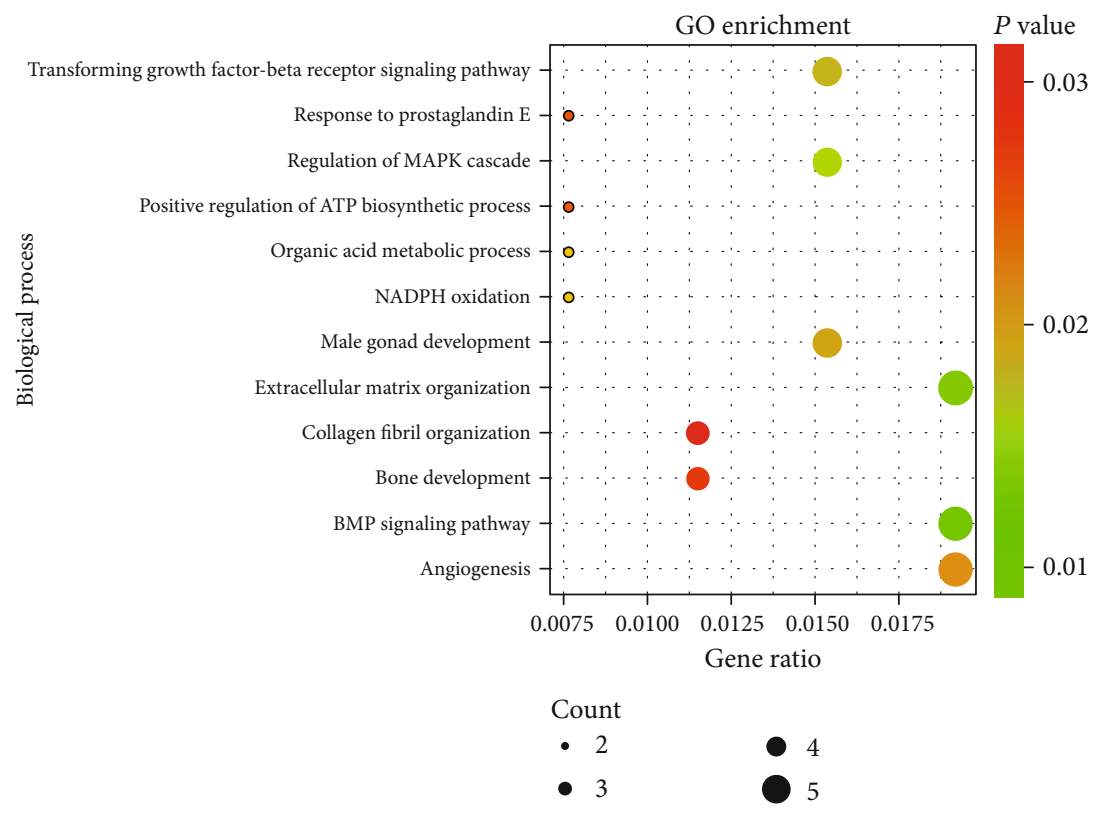

(c)

Figure 2: Continued. 


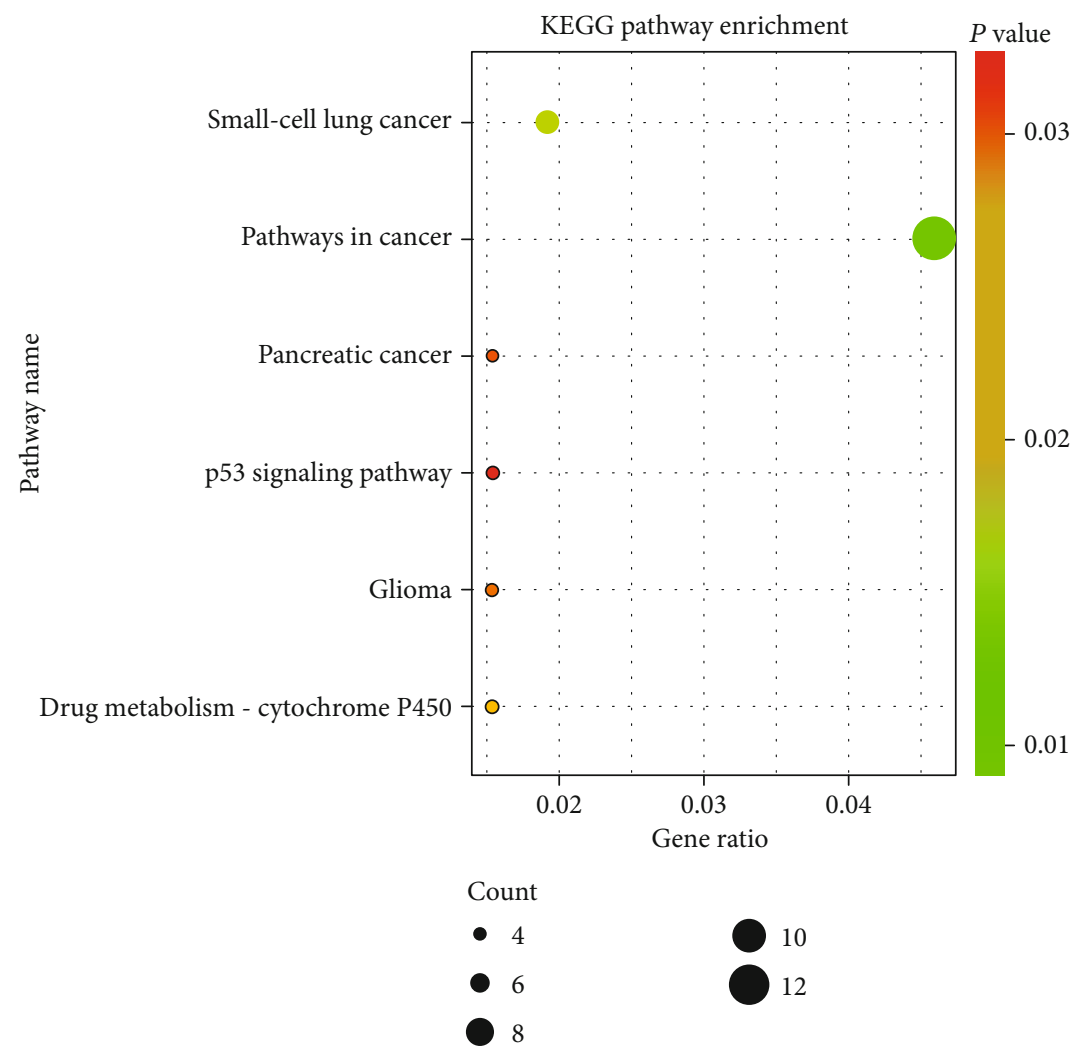

(d)

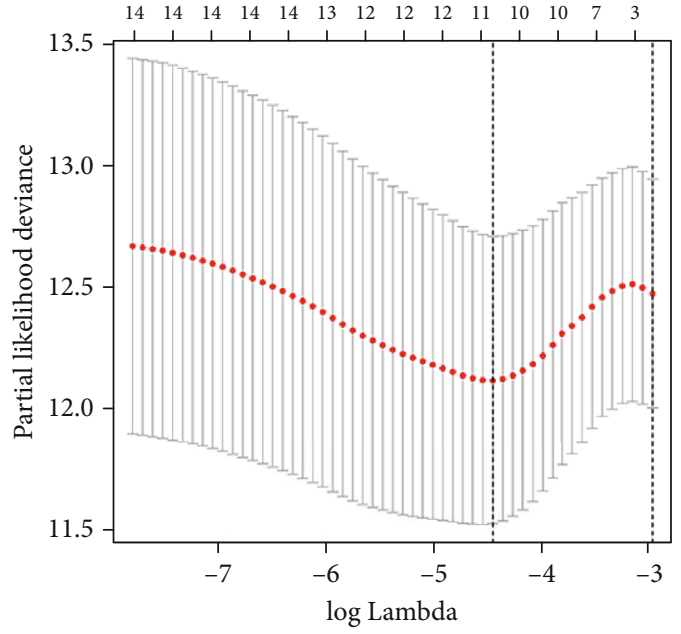

(e)

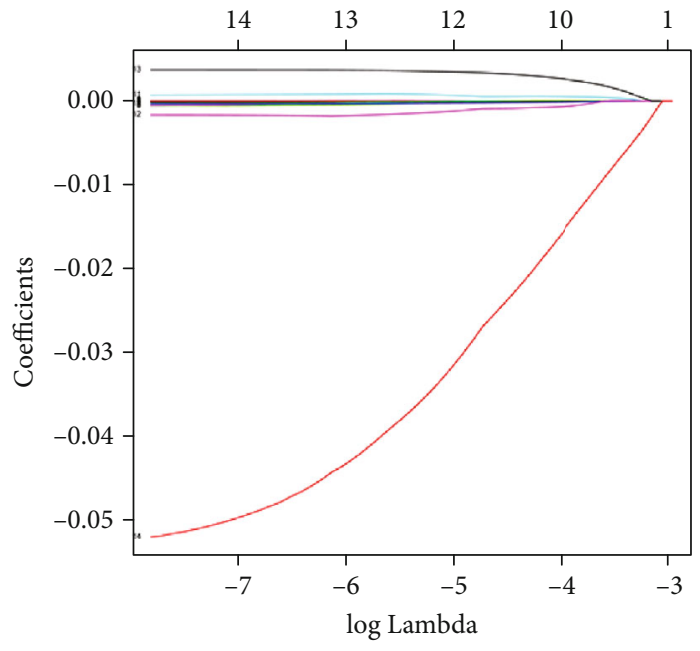

(f)

FIGURE 2: Screening of differentially expressed genes and bioinformatic analysis. $(a, b)$ Venn diagrams show the intersection between genes differentially expressed of the three data sources. (c, d) The dot plot of the enriched biological function and KEGG pathways of the DEGs. (e, f) Lasso regression analysis of the prognostic DEGs.

DEGs and patient DFS data, it was found that a total of 14 DEGs were associated with DFS, including 8 upregulated genes and 6 downregulated genes $(P<0.05)$. For further screening through Lasso regression analysis, 11 genes were selected to perform the fitting between the mRNA and DFS data (Figures 2(e) and 2(f)). The regression coefficient and 5 DEGs obtained through multivariate Cox regression analysis and the expression of the five genes were included in the construction of the model formula to achieve the following risk score formula:

$$
\begin{aligned}
\text { risk score }= & (3.12 \mathrm{E}-05 \times \text { expression value of TENM1 }) \\
& +(3.67 \mathrm{E}-07 \times \text { expression value of FN1 }) \\
& -(5.08 \mathrm{E}-04 \times \text { expression value of APOD }) \\
& +(3.86 \mathrm{E}-03 \times \text { expression value of F12 }) \\
& -(4.76 \mathrm{E}-02 \times \text { expression value of BTNL8) }
\end{aligned}
$$


TABLE 2: Clinical baseline features of DTC patients in the training and testing datasets.

\begin{tabular}{|c|c|c|c|c|}
\hline \multirow[t]{2}{*}{ Clinical features } & \multicolumn{2}{|c|}{$\begin{array}{c}\text { Training dataset } \\
305\end{array}$} & \multicolumn{2}{|c|}{$\begin{array}{c}\text { Testing dataset } \\
128\end{array}$} \\
\hline & $n$ & $\%$ & $n$ & $\%$ \\
\hline \multicolumn{5}{|l|}{ Cancer type, $n(\%)$} \\
\hline PTC & 247 & 80.98 & 104 & 81.25 \\
\hline FTC & 58 & 19.02 & 24 & 18.75 \\
\hline \multicolumn{5}{|l|}{ Age, $n(\%)$} \\
\hline$\leq 55$ & 223 & 73.11 & 88 & 68.75 \\
\hline$>55$ & 82 & 26.89 & 40 & 31.25 \\
\hline \multicolumn{5}{|l|}{ Gender, $n(\%)$} \\
\hline Male & 79 & 25.90 & 39 & 30.47 \\
\hline Female & 226 & 74.10 & 89 & 69.53 \\
\hline Disease-free survival (year) $($ mean $\pm \mathrm{SD})$ & $3.48 \pm 2.84$ & & $2.85 \pm 2.51$ & \\
\hline \multicolumn{5}{|l|}{ Recurrence, $n(\%)$} \\
\hline Yes & 31 & 10.16 & 13 & 10.16 \\
\hline No & 274 & 89.84 & 115 & 89.84 \\
\hline \multicolumn{5}{|l|}{ Tumor size, $n(\%)$} \\
\hline$\leq 2 \mathrm{~cm}$ & 100 & 32.79 & 28 & 21.88 \\
\hline$>2 \mathrm{~cm}$ & 205 & 67.21 & 100 & 78.12 \\
\hline \multicolumn{5}{|l|}{$\mathrm{N}, n(\%)$} \\
\hline N0 & 153 & 50.16 & 70 & 54.69 \\
\hline N1 & 39 & 12.79 & 16 & 12.50 \\
\hline N1a & 65 & 21.31 & 20 & 15.63 \\
\hline N1b & 48 & 15.74 & 22 & 17.18 \\
\hline \multicolumn{5}{|l|}{$\mathrm{M}, n(\%)$} \\
\hline M0 & 182 & 59.67 & 79 & 61.72 \\
\hline M1 & 5 & 1.64 & 2 & 1.56 \\
\hline MX & 118 & 38.69 & 47 & 36.72 \\
\hline \multicolumn{5}{|l|}{ Anatomic site, $n(\%)$} \\
\hline Bilateral & 55 & 18.03 & 28 & 21.88 \\
\hline Isthmus & 13 & 4.26 & 6 & 4.69 \\
\hline Unilateral & 237 & 77.71 & 94 & 73.43 \\
\hline \multicolumn{5}{|l|}{ Stage, $n(\%)$} \\
\hline Stage I & 177 & 58.03 & 70 & 54.69 \\
\hline Stage II & 28 & 9.18 & 14 & 10.94 \\
\hline Stage III & 68 & 22.30 & 27 & 21.09 \\
\hline Stage IV & 32 & 10.49 & 17 & 13.28 \\
\hline \multicolumn{5}{|l|}{ ATA risk stratification, $n(\%)$} \\
\hline High risk & 14 & 4.59 & 8 & 6.25 \\
\hline Intermediate risk & 184 & 60.33 & 67 & 52.34 \\
\hline Low risk & 107 & 35.08 & 53 & 41.41 \\
\hline \multicolumn{5}{|l|}{ ETA risk stratification, $n(\%)$} \\
\hline High risk & 198 & 64.92 & 75 & 58.59 \\
\hline Low risk & 100 & 32.79 & 48 & 37.50 \\
\hline Very low risk & 7 & 2.29 & 5 & 3.91 \\
\hline
\end{tabular}

In the training dataset, the $\mathrm{C}$-index of the five-gene signature is 0.750 (95\% CI: 0.664-0.836), and the 1-year, 3-year, and 5-year AUC were $0.686,0.708$, and 0.659 , respectively. Comparison between the five-gene signature model of this study and other models shows that our model has a good predictive power within training, testing, and entire dataset (Figures 3(a)-3(c)). The ability of the five-gene signature to distinguish between cancer and normal tissue is satisfying, 
1-Year ROC curve of training dataset

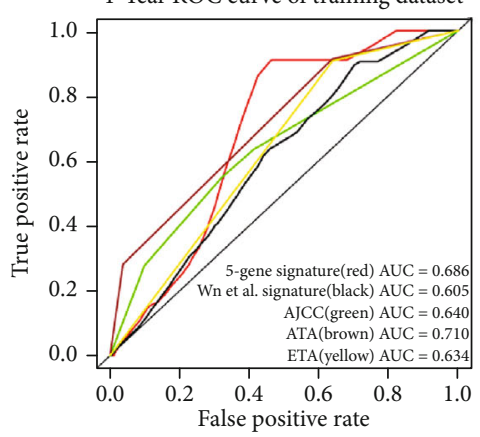

1-Year ROC curve of testing dataset

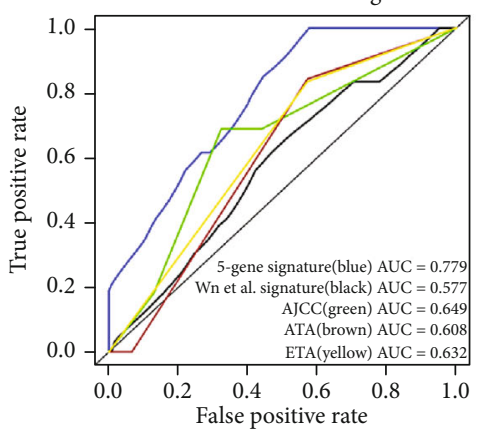

1-Year ROC curve of entire dataset

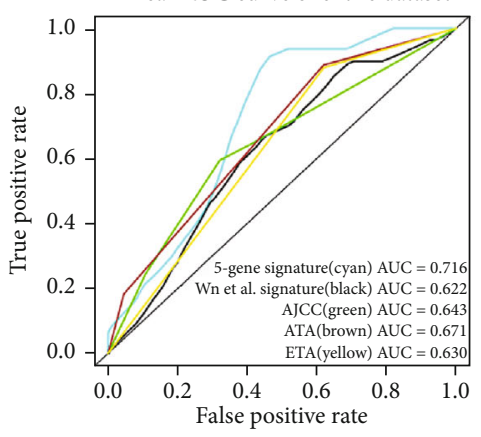

3-Year ROC curve of training dataset

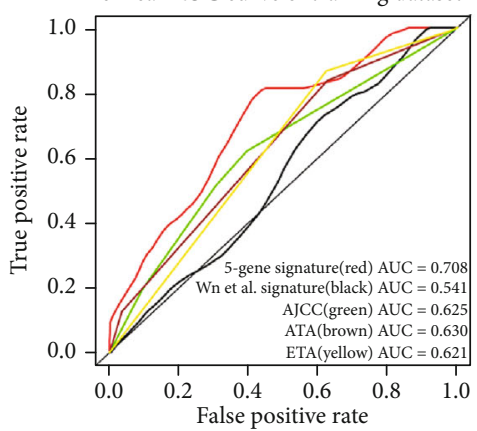

(a)

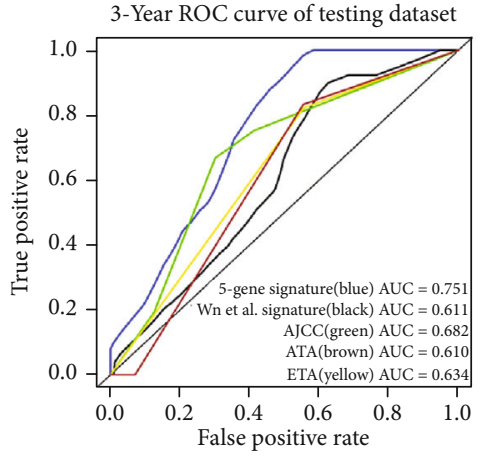

(b)

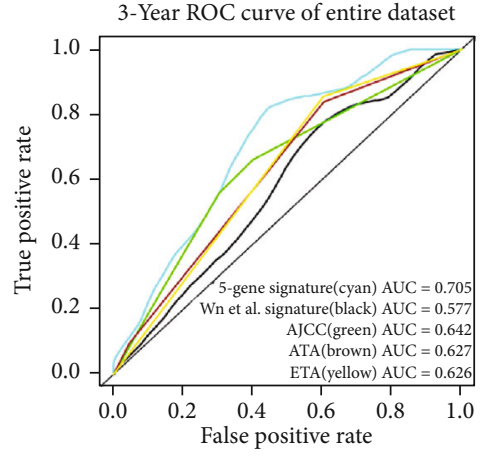

(c)

ROC curve

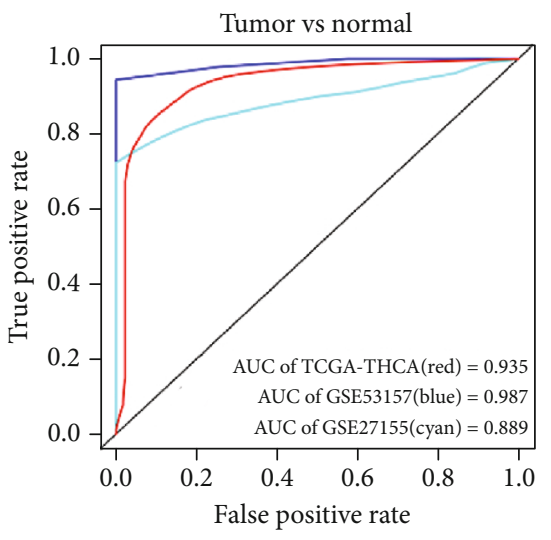

(d)

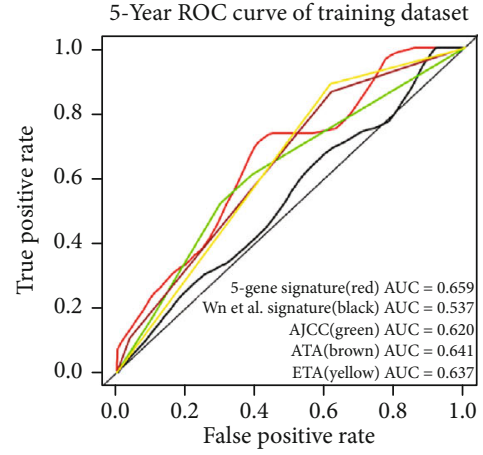

5-Year ROC curve of testing dataset

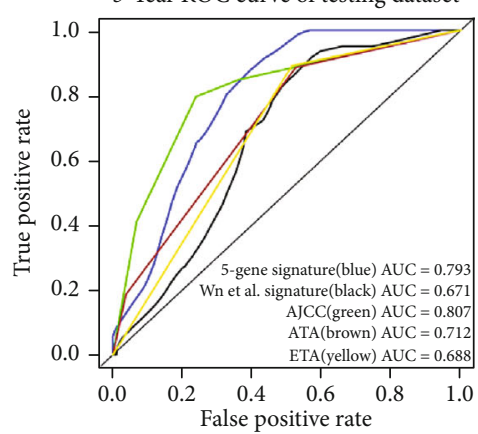

5-Year ROC curve of entire dataset

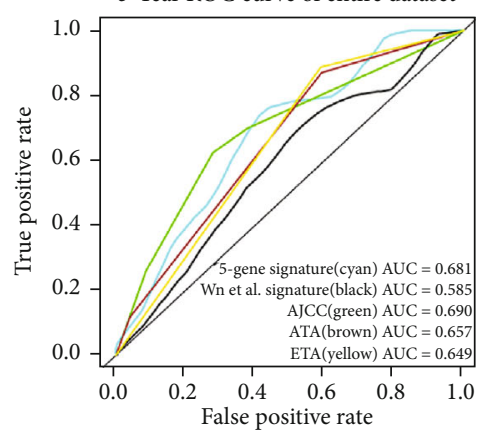

Figure 3: Continued. 


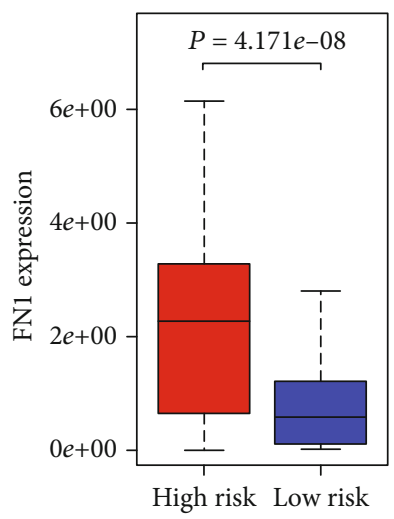

High risk Low risk
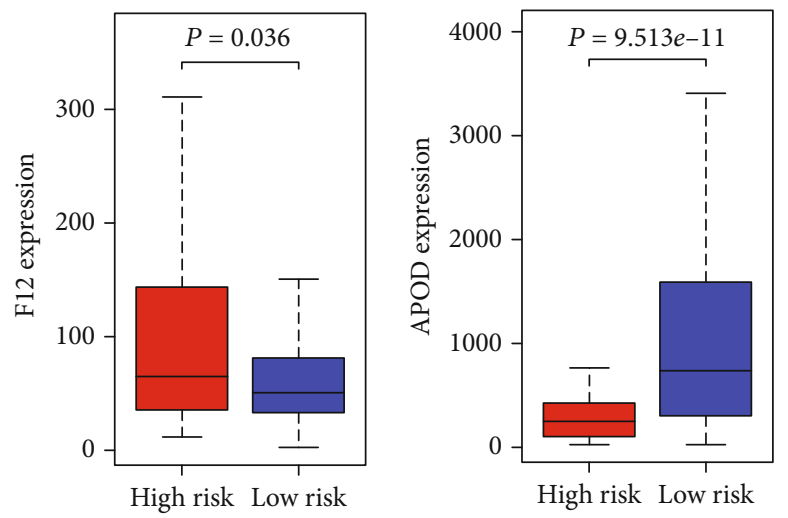

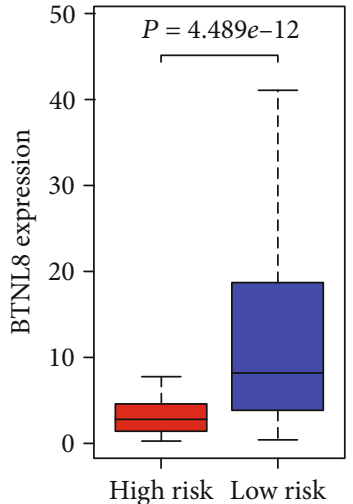

High risk Low risk

(e)
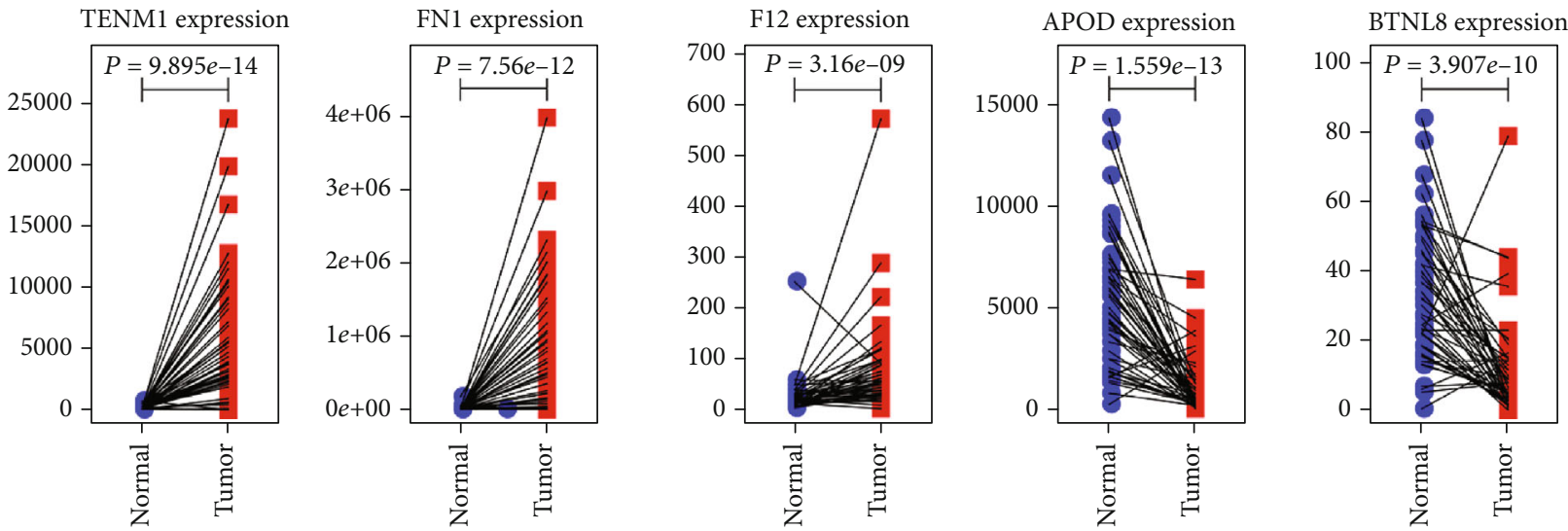

(f)

FIGURE 3: Establishment and validation of the five-gene signature. (a)-(c) ROC curves of the five-gene signature for 1-, 3-, and 5-year DTC patient recurrence compared with other models in the training, testing, and entire dataset. (d) The ability of the five-gene signature to distinguish between cancer and normal tissue in the TCGA, GSE27155, and GSE53157 datasets. (e) The difference of FN1, F12, APOD, and BTNL8 expression levels in the high- and low-risk groups of DTC recurrence patients $(P<0.05)$. (f) The pairing differences between cancerous and paracancerous tissue genes in 58 DTC patients.

since the AUC of the TCGA, GSE27155, and GSE53157 datasets are $0.935,0.889$, and 0.987 , respectively (Figure $3(\mathrm{~d})$ ). The data show that the mRNA expression levels of TENM1, FN1, and F12 are positively correlated with the risk score, while the expression levels of APOD and BTNL8 are negatively correlated with the risk score. Moreover, the expression levels of FN1, F12, APOD, and BTNL8 shows significant differences in the high- and low-risk groups of DTC recurrence $(P<0.05)$ (Figure 3(e)). In addition, according to the pairing differences between cancerous and paracancerous tissue genes in 58 DTC patients, the expression of each gene is significantly different in cancer tissue (Figure 3(f)). We found that TENM1, FN1, and F12 are highly expressed in tumor tissue, while APOD and BTNL8 have a lower expression in tumor tissue. The relationship between the expression of the five genes and clinicopathological characteristics of DTC patients are shown in supplementary figures [21].

3.3. Risk Grouping Analysis on the Five-Gene Signature. The risk score was divided into high- and low-risk groups by applying the $X$-tile software (cutoff value $=0.73$ ). KaplanMeier survival curves of the high- and low-risk group of the training dataset, the testing dataset, and the entire dataset were plotted (Figure 4(a)). All groups have a significant difference in DFS between the high- and low-risk groups $(P<0.001)$. The risk curves, risk scatter plots, and risk distribution heat maps of these three datasets are presented in Figures 4(b)-4(d). GSEA enrichment analysis shows that the P53 signaling pathway, VEGF signaling pathway, thyroid cancer, cell cycle, and DNA replication were more active in the high-risk group when compared to that in the low-risk group $(P<0.01$, FDR $<0.25)$ (Figure $4(\mathrm{e}))$.

3.4. Establishment and Evaluation of a Predictive Nomogram. Univariate Cox regression analysis was performed on the risk scores of the five-gene signature combined with clinical characteristics of the entire dataset (Table 3). Our data show that age ( $>55$ vs. $\leq 55, P=0.004)$, tumor size $(>2 \mathrm{~cm}$ vs. $\leq 2 \mathrm{~cm}, P$ $=0.008), \mathrm{N}$ stage (N1 vs. N0, $P=0.048), \mathrm{M}$ stage (M1 vs. M0 and MX, $P<0.001$ ), AJCC stage (stages III and IV vs. stages I and II, $P=0.001)$, and risk score $(P<0.001)$ were correlated with patient recurrence. In the univariate analysis, the parameters with $P<0.05$ were further incorporated into the multivariate Cox regression analysis. The multivariate Cox regression results indicated that risk score and $M$ stage were independent risk factors for recurrence in DTC patients 

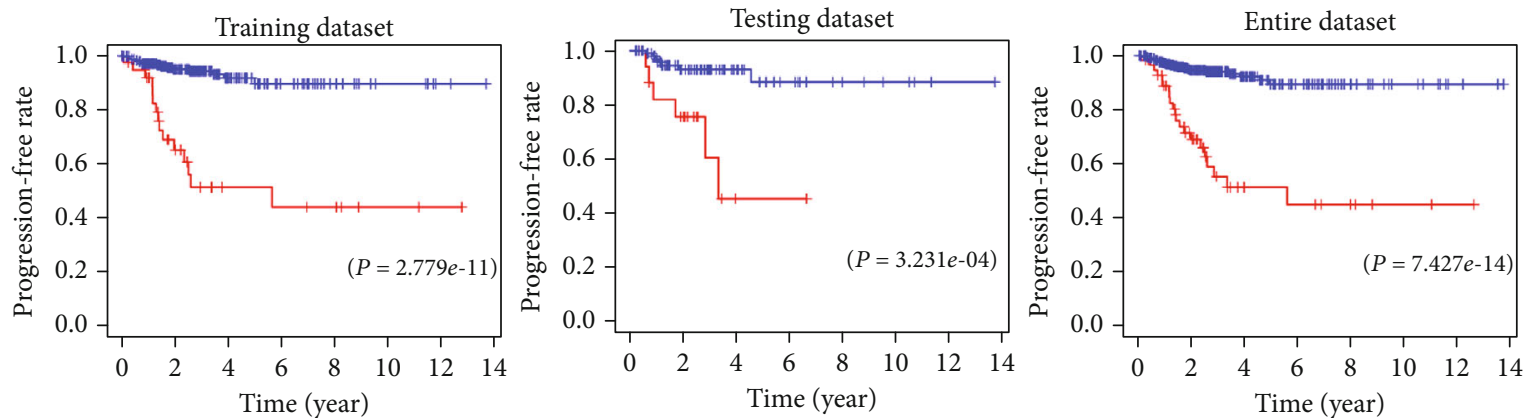

— High risk

— High risk

— Low risk

_ Low risk

— High risk

__ Low risk

(a)
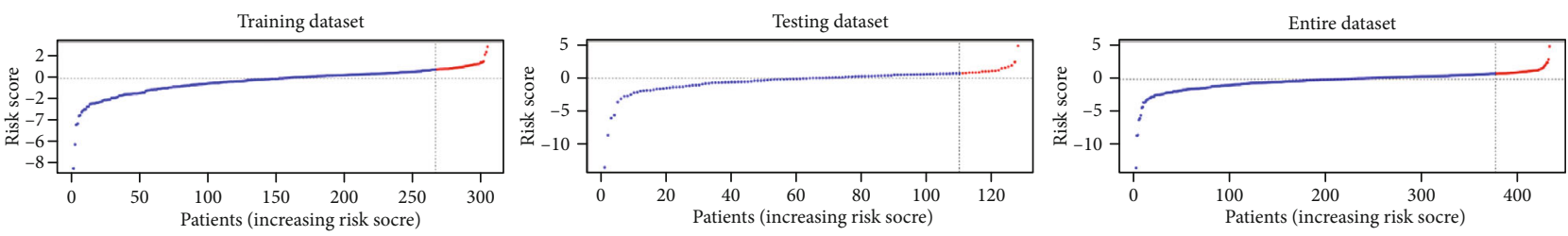

(b)
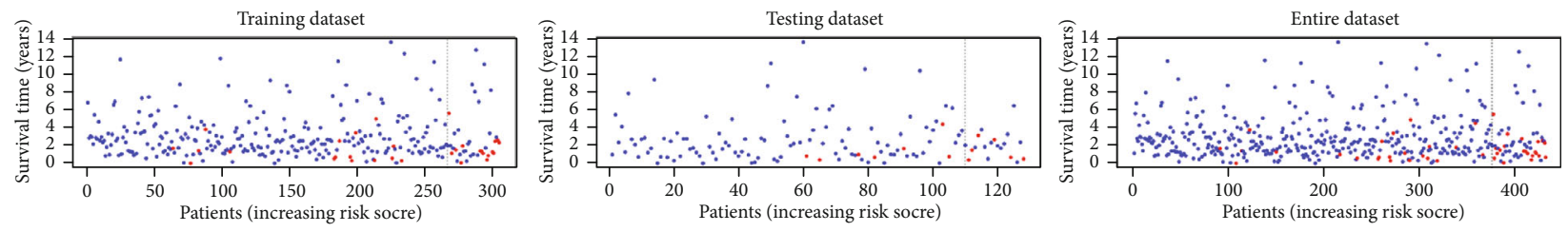

(c)
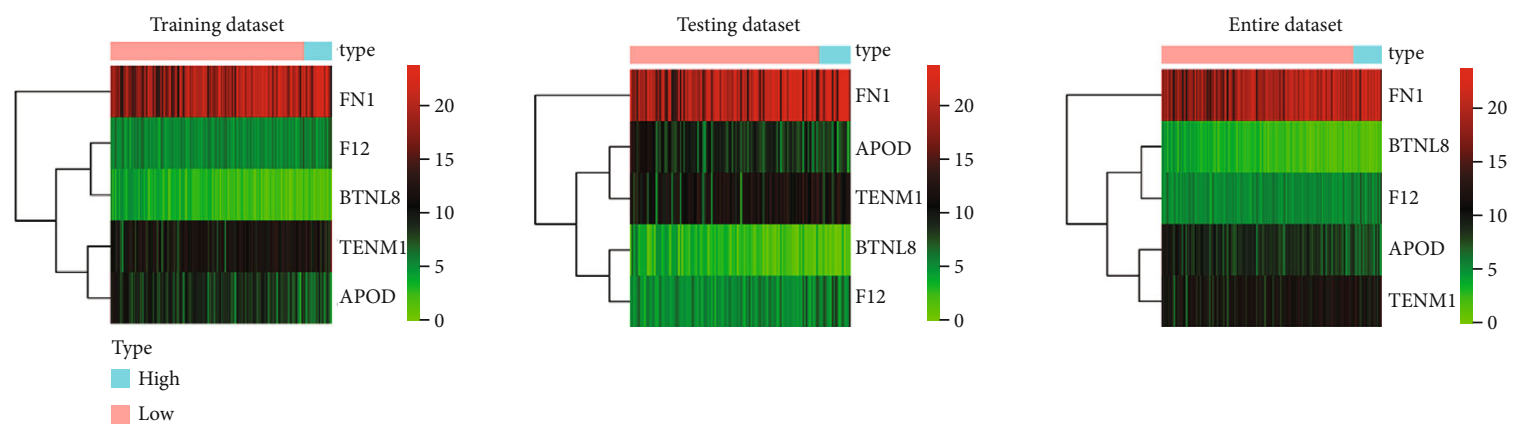

(d)

Figure 4: Continued. 


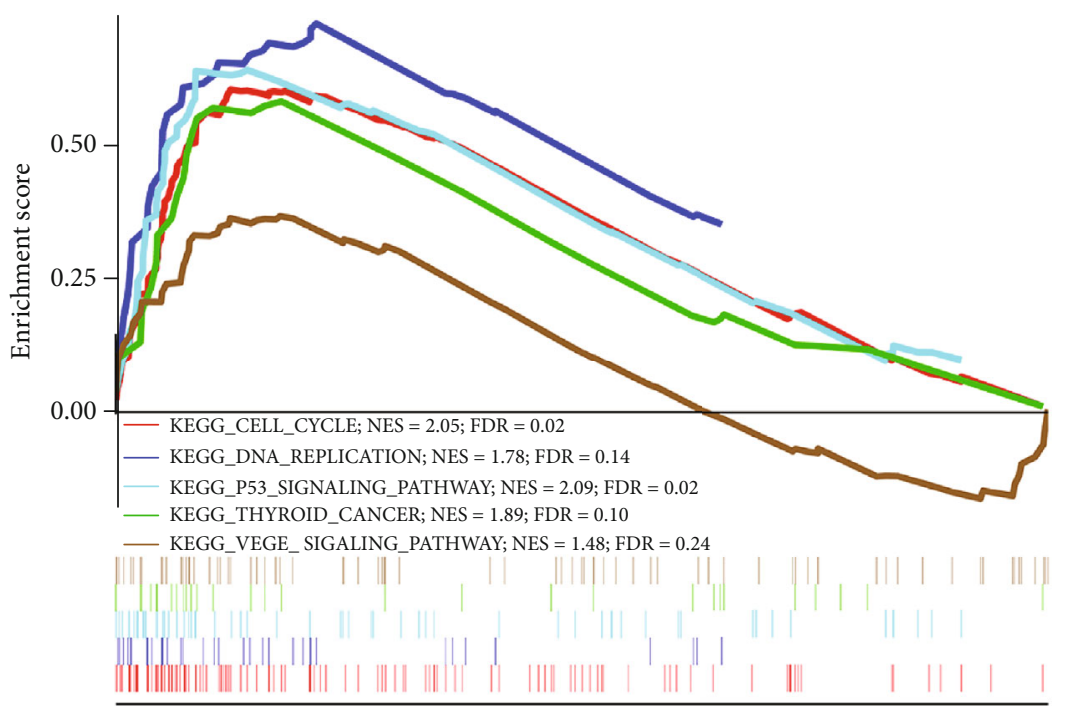

High expression $\leftarrow---------\rightarrow$ Low expression

(e)

FIGURE 4: Risk grouping analysis for the five-gene signature. (a) Kaplan-Meier disease-free survival curve of high- and low-risk group in the training, testing, and entire dataset. (b)-(d) The risk curves, risk scatter plots, and risk distribution heat maps of high- and low-risk group of the training, testing, and entire dataset. (e) GSEA enrichment analysis result map of entire dataset.

TABLE 3: Univariate Cox regression analysis.

\begin{tabular}{|c|c|c|c|}
\hline Clinical features & Statistics & RR (95\% CI) & $P$ value \\
\hline Risk score $($ mean $\pm S D)$ & $-0.396 \pm 1.471$ & $2.436(1.900-3.123)$ & $<0.001$ \\
\hline \multicolumn{4}{|l|}{ Cancer type, $n(\%)$} \\
\hline PTC & 351 & 1 & \\
\hline FTC & 82 & $0.713(0.301-1.687)$ & 0.441 \\
\hline \multicolumn{4}{|l|}{ Age, $n(\%)$} \\
\hline$\leq 55$ & 311 & 1 & \\
\hline$>55$ & 122 & $2.394(1.320-4.341)$ & 0.004 \\
\hline \multicolumn{4}{|l|}{ Gender, $n(\%)$} \\
\hline Male & 118 & 1 & \\
\hline Female & 315 & $0.772(0.409-1.456)$ & 0.423 \\
\hline \multicolumn{4}{|l|}{ Tumor size, $n(\%)$} \\
\hline$\leq 2 \mathrm{~cm}$ & 128 & 1 & \\
\hline$>2 \mathrm{~cm}$ & 305 & $4.046(1.446-11.317)$ & 0.008 \\
\hline \multicolumn{4}{|l|}{$\mathrm{N}$ stage, $n(\%)$} \\
\hline No & 223 & 1 & \\
\hline N1 & 210 & $1.856(1.004-3.431)$ & 0.048 \\
\hline \multicolumn{4}{|l|}{ M stage, $n(\%)$} \\
\hline M0 and MX & 426 & 1 & \\
\hline M1 & 7 & $6.692(2.375-18.853)$ & $<0.001$ \\
\hline \multicolumn{4}{|l|}{ AJCC stage, $n(\%)$} \\
\hline Stages I and II & 289 & 1 & \\
\hline Stages III and IV & 144 & $2.746(1.515-4.977)$ & 0.001 \\
\hline \multicolumn{4}{|l|}{ Anatomic site, $n(\%)$} \\
\hline Unilateral & 331 & 1 & \\
\hline Isthmus & 19 & $0.469(0.064-3.427)$ & 0.455 \\
\hline Bilateral & 83 & $1.258(0.599-2.641)$ & 0.544 \\
\hline
\end{tabular}


TABle 4: Multivariate Cox regression analysis.

\begin{tabular}{|c|c|c|c|c|c|c|}
\hline \multirow{2}{*}{ Clinical features } & \multicolumn{2}{|l|}{ Nonadjusted } & \multicolumn{2}{|l|}{ Adjust I } & \multicolumn{2}{|l|}{ Adjust II } \\
\hline & $\mathrm{RR}(95 \% \mathrm{CI})$ & $P$ value & RR (95\% CI) & $P$ value & RR (95\% CI) & $P$ value \\
\hline Risk score $($ mean $\pm \mathrm{SD})$ & $2.171(1.626-2.898)$ & $<0.001$ & $2.148(1.620-2.849)$ & $<0.0001$ & $2.169(1.636-2.874)$ & $<0.0001$ \\
\hline \multicolumn{7}{|l|}{ Cancer type, $n(\%)$} \\
\hline PTC & 1 & & NA & NA & NA & NA \\
\hline FTC & $1.000(0.392-2.552)$ & 1.000 & & & & \\
\hline \multicolumn{7}{|l|}{ Age, $n(\%)$} \\
\hline$\leq 55$ & 1 & & 1 & & 1 & \\
\hline$>55$ & $1.367(0.579-3.228)$ & 0.476 & $1.336(0.580-3.079)$ & 0.496 & $1.584(0.811-3.093)$ & 0.178 \\
\hline \multicolumn{7}{|l|}{ Gender, $n(\%)$} \\
\hline Male & 1 & & NA & NA & NA & NA \\
\hline Female & $1.231(0.619-2.450)$ & 0.553 & & & & \\
\hline \multicolumn{7}{|l|}{ Tumor size, $n(\%)$} \\
\hline$\leq 2 \mathrm{~cm}$ & 1 & & 1 & & 1 & \\
\hline$>2 \mathrm{~cm}$ & $2.306(0.784-6.784)$ & 0.129 & $2.339(0.804-6.804)$ & 0.119 & $2.444(0.847-7.054)$ & 0.098 \\
\hline \multicolumn{7}{|l|}{$\mathrm{N}, n(\%)$} \\
\hline No & 1 & & 1 & & 1 & \\
\hline N1 & $1.673(0.837-3.344)$ & 0.145 & $1.644(0.860-3.143)$ & 0.133 & $1.739(0.927-3.261)$ & 0.085 \\
\hline \multicolumn{7}{|l|}{$\mathrm{M}, n(\%)$} \\
\hline M0 and MX & 1 & & 1 & & 1 & \\
\hline M1 & $4.576(1.523-13.755)$ & 0.0274 & $4.411(1.521-12.788)$ & 0.006 & $4.478(1.546-12.967)$ & 0.006 \\
\hline \multicolumn{7}{|l|}{ Stage, $n(\%)$} \\
\hline Stages I and II & 1 & & 1 & & NA & NA \\
\hline Stages III and IV & $1.287(0.569-2.908)$ & 0.545 & $1.313(0.586-2.943)$ & 0.508 & & \\
\hline \multicolumn{7}{|l|}{ Anatomic site, $n(\%)$} \\
\hline Unilateral lobe & 1 & & NA & NA & NA & NA \\
\hline Isthmus & $0.689(0.091-5.183)$ & 0.717 & & & & \\
\hline Bilateral lobe & $1.354(0.628-2.916)$ & 0.439 & & & & \\
\hline
\end{tabular}

Adjust I model adjusts for parameters with $P$ value $<0.05$ based on univariate Cox regression analysis. Adjust II model adjusts for risk score, age, tumor size, $\mathrm{N}$, and $M$.

$(P<0.05)$ (Table 4$)$. The $\mathrm{C}$-index of the nomogram was 0.797 (95\% CI: 0.730-0.864). The 1-year, 3-year, and 5-year ROC curves of the nomogram model were established (Figure 5(a)), and the AUC results demonstrated that the accuracy of the nomogram prediction was superior to other models (Figure 5(b)). Moreover, calibration curve analysis was performed on the nomogram model (Figure 5(c)).

\section{Discussion}

Thyroid cancer is the common endocrine malignancy that occurs in the head and neck, of which the pathogenesis is poorly understood [1]. Exposure to radiation, iodine intake, genetics, and other factors can cause thyroid cancer [1]. Even after patients have undergone standard surgery, ${ }^{131} \mathrm{I}$ and thyroid hormone suppression therapy, $5 \%-23 \%$ of patients still suffer from metastatic recurrence [22]. A good prediction model of patient prognosis can effectively identify patients with high risk of recurrence and foster their individualized indepth treatment to achieve better therapeutic outcomes. The combined prediction of various prognostic markers derived from gene expression profiling can reflect the prog- nosis of individual patients at the molecular level. Such a gene signature can be complementary with traditional AJCC, ATA, and ETA staging prediction systems.

In this study, GO biological process enrichment analysis and KEGG pathway enrichment analysis were performed using cancer-related DEGs, which were derived from the intersection between the TCGA-THCA, GSE27155, and GSE53157 datasets. The results showed that transforming growth factor-beta receptor signaling pathway, BMP signaling pathway, regulation of MAPK cascade, and extracellular matrix organization are significantly enriched in the analysis of biological processes. Among the extracellular matrix proteins, collagen, fibronectin, and integrin represent the major components. Some studies indicate that type IV collagenase and matrix metalloproteinases secreted by thyroid cancer cells can promote the metastasis of cancer cells by destroying the extracellular matrix structure, which affects the prognosis of thyroid cancer [23-25]. Fibronectin and integrin can participate in the adhesion process of tumor cells and extracellular matrix through activation of the Ras/Raf/Mek pathway and the calmodulin dependent kinase-II (CaMKII) pathway [26]. Transforming growth factor-beta 1 (TGF- $\beta 1$ ) and 
Points

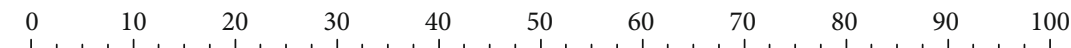

Age
$\mathrm{M}$
Tumor size
Risk score
Total points
1-year Disease-free survival
3-year Disease-free survival
5-year Disease-free survival
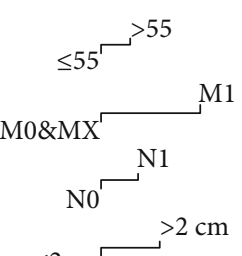

\begin{tabular}{|c|c|c|c|c|c|c|c|c|c|c|c|c|}
\hline-14 & -12 & -10 & -8 & & -6 & & -4 & -2 & 0 & 2 & 4 & 6 \\
\hline 0 & 10 & 20 & 30 & 40 & & 50 & 60 & 70 & 80 & 90 & 100 & 110 \\
\hline
\end{tabular}

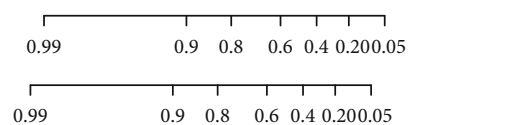

(a)
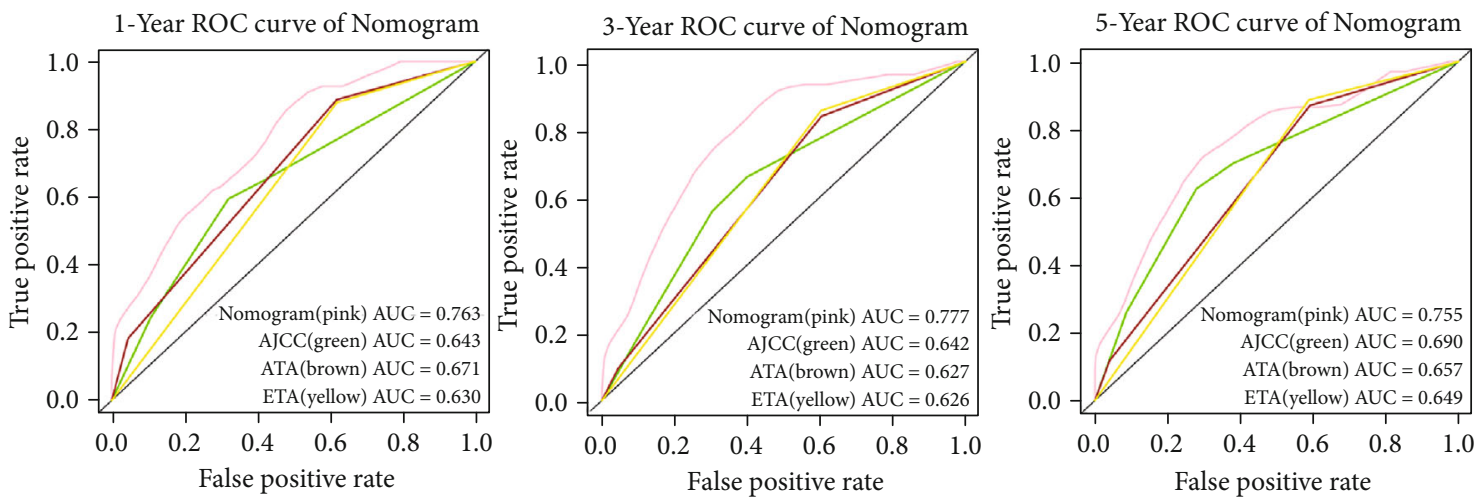

(b)
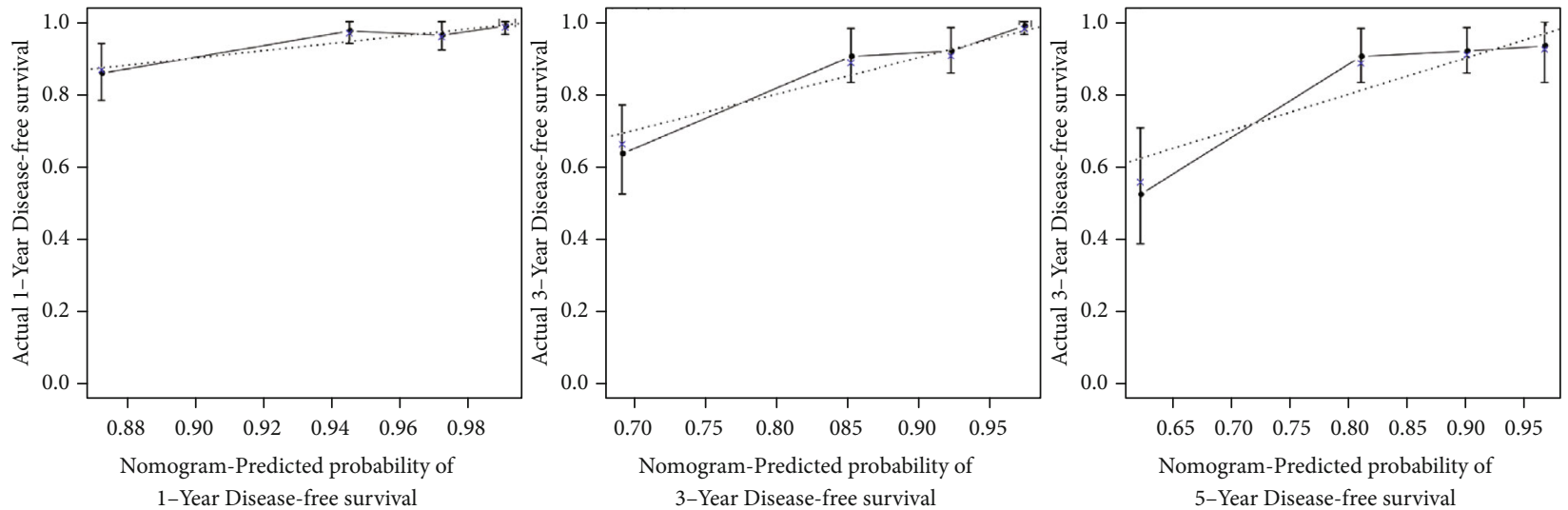

(c)

FIGURE 5: Establishment and validation of a predictive nomogram. (a) The nomogram for predicting proportion of DTC patients with 1-, 3-, and 5-year disease-free survival. (b) ROC curves of the nomogram for 1-, 3-, and 5-year DTC patients recurrence compared with other models in the entire dataset. (c) The calibration curve of the nomogram.

BMP-2 are members of the transforming growth factor-beta family, which exert important roles in tumor growth and invasion. TGF- $\beta 1$ and epidermal growth factor- (EGF-) like ligands have opposite roles in different stages of thyroid cancer growth: on one hand, they act as tumor suppressors through inhibiting the proliferation of thyroid cells and reg- ulating the formation of extracellular matrix [27, 28]; on the other hand, they can promote angiogenesis in advanced stage of thyroid cancer [29]. TGF $\beta$ and BMPs pathways can contribute to the synergistic suppression of tumor growth, but atypical TGF $\beta$ that activates PI3K/AKT signaling can reverse this suppression and promote tumor cell proliferation 
[30]. The mitogen-activated protein kinase (MAPK) pathway is one of the most classical signal transduction pathways in thyroid cancer. In this pathway, BRAF-mutations and RET/PTC rearrangements can promote the transformation of thyroid follicular cells into papillary thyroid cancer, which is considered as a hallmark for PTC development and progression [31].

The five-gene signature was established as a new molecular predictive index for the recurrence in DTC patients. The results of multivariate Cox regression coefficient of the five genes indicated that TENM1, FN1, and F12 were protective factors, while APOD and BTNL8 were unfavorable elements for recurrence risk of DTC patients. Multiple studies have shown that the expression of TENM1, which acts as a cell signal transducer in neurons, is highly positively correlated with the growth and invasion of PTC, and it is a potential biomarker for early diagnosis of thyroid cancer [32-34]. FN1 encodes fibronectin and is involved in cell proliferation, adhesion, and migration. Previous studies demonstrated that FN1 is a potential therapeutic target highly related to tumor invasion in PTC and medullary carcinoma, and it is also one of the markers to distinguish malignant from benign nodules in thyroid cancer [35-37]. F12 encodes coagulation factor XII which circulates in blood as a zymogen; however, the relationship between F12 and tumorigenesis has not been reported. This study pointed out that F12 is highly expressed in DTC tissues. Furthermore, our results demonstrated that the F12 expression is higher in FTC tissues than in PTC tissues, and its expression is highly correlated with patient age, lymph node metastasis, and postoperative recurrence risk $(P<0.05)$. APOD, known as apolipoprotein $\mathrm{D}$, is regulated by P73 and P63 proteins, which belong to the P53 tumor suppressor family [38]. Moreover, it has been shown that APOD can inhibit the proliferation of cancer cells in breast cancer, prostate cancer, and colorectal cancer cell lines, and it may be used as a marker of the initial stage of tumor deterioration [39-41]. Butyrophilin-like (BTNL) protein can regulate $\mathrm{T}$ lymphocyte response. Research indicates that BTNL8 is also related to inflammatory disorders and tumorigenesis. In intestinal tumors, where BTNL8 was found to be downregulated, it can enhance the immune response mediated by $\mathrm{T}$ cells and plays a role in immune surveillance of tumor cells [42].

In this study, we established a high- and low-risk group (cutoff value $=0.73$ ) of a five-gene signature through $X$-tile software. GSEA enrichment analysis of the two groups showed that the P53 signaling pathway and the VEGF signaling pathway were significantly enriched in the high-risk group. The P53 signaling pathway plays a central role in tumorigenesis. TP53 is one of the most important tumor suppressor genes, which has the functions of inhibiting cell proliferation, participating in cell cycle regulation and inducing cell apoptosis. Due to a short protein half-life, wild-type P53 is expressed at a low level in cells, whereas mutant (inactive) P53 is frequently overexpressed in cancer cells due to its higher stability. Therefore, the high expression of the P53 protein in the tumor tissue is closely related to poor prognosis [43]. Vascular endothelial growth factor (VEGF) is an essential factor for the development of blood vessels, which is crucial for the growth of tumors. Lymphatic vessel density and VEGF-C expression have been found to be significantly different between benign and malignant tissue [44]. The expression of VEGF is highly correlated with the tumorigenesis and the prognosis of DTC $[45,46]$. The abovementioned pathway analysis showed that the biological processes related to cancer growth was enriched in the high-risk group. The Kaplan-Meier survival correlation curve of the training dataset and the testing dataset intuitively reflected the difference in DFS rates of the two groups, suggesting that the cutoff points of the high- and low-risk groups were well differentiated.

In many studies, nomograms for predicting the recurrence of thyroid cancer patients had been previously formulated. Pathak et al. established a 10-year recurrence prediction nomogram (C-index 0.76) for thyroid cancer patients [47]. Ding et al. successfully established a 3-, 5-, and 10-year cancer recurrence prediction nomogram (Cindex 0.70) for PTC patients [48]. Wu et al. constructed a prognostic nomogram related to five-gene signature by analyzing the data from GEO and TCGA, with the goal to predict progression-free interval of PTC patients. The C-index of the model is 0.76 , which has good predictive value for PTC [11]. However, a relevant prognostic nomogram for DTC patients has not been developed so far. In this study, we found that the risk score and $M$ stage were independent risk factors for recurrence in DTC patients $(P<0.05)$, and we established a 3-, 5-, and 10-year cancer recurrence prediction nomogram (C-index 0.80) for DTC patients.

Although this study segmented $30 \%$ of the data from the overall population for validation, a large sample validation analysis of other cohorts could not be carried out due to the limited availability of data. Since in this study, the clinical characteristics of patients that could be included in the analysis was limited, and more indepth verification is warranted. It is worth noting that this study is the first analyzing the recurrence prediction of DTC patients from the level of the prognostic related gene expression, and its accuracy and individualized prediction ability were more prominent compared to that of traditional clinical feature prediction models.

In conclusion, we have successfully established a nomogram of a five-gene signature combined with traditional clinical characteristics to predict the 1-year, 3-year, and 5-year DFS rate of DTC patients, which can be used as a tool to assess patients' postoperative recurrence rate and to formulate an individualized, accurate treatment strategy.

\section{Data Availability}

The data used to support the findings of this study have been deposited in the figshare repository: (doi:10.6084/m9.figshare .11734497.v2; doi:10.6084/m9.figshare.11734500.v2; doi:10 .6084/m9.figshare.11734494.v2).

\section{Conflicts of Interest}

All authors confirm that there are no conflicts of interest. 


\section{Acknowledgments}

We thank all staff for their help and support in the study. This research was funded by the Chongqing Higher Education Teaching Reform Research Project, grant number: 193385.

\section{References}

[1] S. I. Sherma, "Thyroid carcinoma," The Lancet, vol. 361, no. 9356, pp. 501-511, 2003.

[2] L. Davies and H. G. Welch, "Current thyroid cancer trends in the United States," JAMA Otolaryngology- Head \& Neck Surgery, vol. 140, no. 4, pp. 317-322, 2014.

[3] E. L. Mazzaferri and R. T. Kloos, "Clinical review 128: current approaches to primary therapy for papillary and follicular thyroid cancer," The Journal of Clinical Endocrinology and Metabolism, vol. 86, no. 4, pp. 1447-1463, 2001.

[4] C. Durante, T. Montesano, M. Torlontano et al., "Papillary thyroid cancer: time course of recurrences during postsurgery surveillance," The Journal of Clinical Endocrinology and Metabolism, vol. 98, no. 2, pp. 636-642, 2013.

[5] C. S. Grant, I. D. Hay, I. R. Gough, E. J. Bergstralh, J. R. Goellner, and W. McConahey, "Local recurrence in papillary thyroid carcinoma: is extent of surgical resection important?," Surgery, vol. 104, no. 6, pp. 954-962, 1988.

[6] M. B. Amin, F. L. Greene, S. B. Edge et al., "The eighth edition AJCC Cancer staging manual: continuing to build a bridge from a population-based to a more "personalized" approach to cancer staging," CA: A Cancer Journal for Clinicians, vol. 67, no. 2, pp. 93-99, 2017.

[7] B. Haugen, "2015 American Thyroid Association Management Guidelines for adult patients with thyroid nodules and differentiated thyroid cancer: what is new and what has changed?," Cancer, vol. 123, no. 3, pp. 372-381, 2017.

[8] G. Russ, S. Bonnema, M. Erdogan, C. Durante, R. Ngu, and L. Leenhardt, "European thyroid association guidelines for ultrasound malignancy risk stratification of thyroid nodules in adults: the EU-TIRADS," European Thyroid Journal, vol. 6, no. 5, pp. 225-237, 2017.

[9] X. Tian, X. Zhu, T. Yan et al., "Differentially expressed lncRNAs in gastric cancer patients: a potential biomarker for gastric cancer prognosis," Journal of Cancer, vol. 8, no. 13, pp. 2575-2586, 2017.

[10] M. Wu, X. Li, T. Zhang, Z. Liu, and Y. Zhao, "Identification of a nine-gene signature and establishment of a prognostic nomogram predicting overall survival of pancreatic cancer," Frontiers in Oncology, vol. 9, p. 996, 2019.

[11] M. Wu, H. Yuan, X. Li, Q. Liao, and Z. Liu, "Identification of a five-gene signature and establishment of a prognostic nomogram to predict progression-free interval of papillary thyroid carcinoma," Frontiers in Endocrinology, vol. 10, p. 790, 2019.

[12] A. Iasonos, D. Schrag, G. V. Raj, and K. S. Panageas, "How to build and interpret a nomogram for cancer prognosis," Journal of Clinical Oncology, vol. 26, no. 8, pp. 1364-1370, 2008.

[13] W. Liang, L. I. Zhang, G. Jiang et al., "Development and validation of a nomogram for predicting survival in patients with resected non-small-cell lung cancer," Journal of Clinical Oncology, vol. 33, no. 8, pp. 861-869, 2015.

[14] Y. Q. Huang, C. H. Liang, L. He et al., "development and validation of a radiomics nomogram for preoperative prediction of lymph node metastasis in colorectal cancer," Journal of Clinical Oncology, vol. 34, no. 18, pp. 2157-2164, 2016.

[15] W. Xiang, Clinical featuresof DTC patients from the TCGATHCA data set, figshare. Dataset, 2020.

[16] N. D. Perrier, J. D. Brierley, and R. M. Tuttle, "Differentiated and anaplastic thyroid carcinoma: major changes in the American joint committee on Cancer eighth edition cancer staging manual," CA: A Cancer Journal for Clinicians, vol. 68, no. 1, pp. 55-63, 2018.

[17] W. Xiang, The training dataset, figshare. Dataset, 2020.

[18] W. Xiang, The testing dataset, figshare. Dataset, 2020.

[19] R. L. Camp, M. Dolled-Filhart, and D. L. Rimm, "X-Tile," Clinical Cancer Research, vol. 10, no. 21, pp. 7252-7259, 2004.

[20] N. R. Clark and A. Ma'ayan, "Introduction to statistical methods for analyzing large data sets: gene-set enrichment analysis," Science Signaling, vol. 4, no. 190, p. tr4, 2011.

[21] W. Xiang, The relationship between the expression of the five genes and clinicopathological characteristics of DTC patients, figshare. Figure, 2020.

[22] M. Haq and C. Harmer, "Differentiated thyroid carcinoma with distant metastases at presentation: prognostic factors and outcome," Clinical Endocrinology, vol. 63, no. 1, pp. 8793, 2005.

[23] T. Kusunoki, S. Nishida, S. Kimoto-Kinoshita, K. Murata, T. Satou, and T. Tomura, "Type IV collagen, type IV collagenase activity and ability of cell proliferation in human thyroid tumours," Asian Journal of Surgery, vol. 25, no. 4, pp. 304-308, 2002.

[24] H. Tan, K. Ye, Z. Wang, and H. Tang, "Clinicopathologic evaluation of immunohistochemical CD147 and MMP-2 expression in differentiated thyroid carcinoma," Japanese Journal of Clinical Oncology, vol. 38, no. 8, pp. 528-533, 2008.

[25] M. Yeh, J. Rougier, J. Park et al., "Differentiated thyroid cancer cell invasion is regulated through epidermal growth factor receptor-dependent activation of matrix metalloproteinase (MMP)-2/gelatinase A," Endocrine-Related Cancer, vol. 13, no. 4, pp. 1173-1183, 2006.

[26] M. Illario, A. Cavallo, S. Monaco et al., "Fibronectin-induced proliferation in thyroid cells is mediated by alphavbeta 3 integrin through Ras/Raf-1/MEK/ERK and calcium/CaMKII signals," The Journal of Clinical Endocrinology \& Metabolism, vol. 90, no. 5, pp. 2865-2873, 2005.

[27] B. Grubeck-Loebenstein, G. Buchan, R. Sadeghi et al., "Transforming growth factor beta regulates thyroid growth. Role in the pathogenesis of nontoxic goiter," The Journal of Clinical Investigation, vol. 83, no. 3, pp. 764-770, 1989.

[28] C. Garbi, G. Colletta, A. M. Cirafici, P. C. Marchisio, and L. Nitsch, "Transforming growth factor-beta induces cytoskeleton and extracellular matrix modifications in FRTL-5 thyroid epithelial cells," European Journal of Cell Biology, vol. 53, no. 2, pp. 281-289, 1990.

[29] G. Mincione, M. C. di Marcantonio, C. Tarantelli et al., "EGF and TGF- ? 1 Effects on Thyroid Function," Journal of Thyroid Research, vol. 2011, Article ID 431718, 13 pages, 2011.

[30] F. Dituri, C. Cossu, S. Mancarella, and G. Giannelli, “The interactivity between TGF $\beta$ and BMP signaling in organogenesis, fibrosis, and cancer," Cells, vol. 8, no. 10, p. 1130, 2019.

[31] R. Witt, R. Ferris, E. Pribitkin, S. Sherman, D. Steward, and Y. Nikiforov, "Diagnosis and management of differentiated thyroid cancer using molecular biology," The Laryngoscope, vol. 123, no. 4, pp. 1059-1064, 2013. 
[32] S.-P. Cheng, M.-J. Chen, M.-N. Chien, C.-H. Lin, J.-J. Lee, and C.-L. Liu, "Overexpression of teneurin transmembrane protein 1 is a potential marker of disease progression in papillary thyroid carcinoma," Clinical and Experimental Medicine, vol. 17, no. 4, pp. 555-564, 2017.

[33] Y. Sun, Z. Liu, B. Yang, and B. Yu, "MicroRNA-486 inhibits cell proliferation, invasion and migration via downregulating the TENM1 expressions and affecting ERK and Akt signaling pathways and epithelial-to-mesenchymal transition in papillary thyroid carcinoma," European Review for Medical Pharmacological Sciences, vol. 23, no. 19, pp. 84298439, 2019.

[34] D. Wu, S. Hu, Y. Hou, Y. He, and S. Liu, "Identification of potential novel biomarkers to differentiate malignant thyroid nodules with cytological indeterminate," BMC Cancer, vol. 20, no. 1, p. 199, 2020.

[35] Q. Wang, E. Chen, Y. Cai et al., "A panel of four genes accurately differentiates benign from malignant thyroid nodules," Journal of Experimental Clinical Cancer Research, vol. 35, no. 1, p. 169, 2016.

[36] S. Zhan, J. Li, T. Wang, and W. Ge, "Quantitative proteomics analysis of sporadic medullary thyroid cancer reveals FN1 as a potential novel candidate prognostic biomarker," The Oncologist, vol. 23, no. 12, pp. 1415-1425, 2018.

[37] M. Sponziello, F. Rosignolo, M. Celano et al., "Fibronectin-1 expression is increased in aggressive thyroid cancer and favors the migration and invasion of cancer cells," Molecular and Cellular Endocrinology, vol. 431, pp. 123-132, 2016.

[38] Y. Sasaki, H. Negishi, R. Koyama et al., "p53 family members regulate the expression of the apolipoprotein $\mathrm{D}$ gene," The Journal of Biological Chemistry, vol. 284, no. 2, pp. 872-883, 2009.

[39] P. Provost, Y. Marcel, R. Milne, P. Weech, and E. Rassart, "Apolipoprotein D transcription occurs specifically in nonproliferating quiescent and senescent fibroblast cultures," FEBS Letters, vol. 290, no. 1-2, pp. 139-141, 1991.

[40] Y. López-Boado, J. Tolivia, and C. López-Otín, “Apolipoprotein $\mathrm{D}$ gene induction by retinoic acid is concomitant with growth arrest and cell differentiation in human breast cancer cells.," The Journal of Biological Chemistry, vol. 269, no. 43, pp. 26871-26878, 1994.

[41] R. Bajo-Grañeras, J. Crespo-Sanjuan, R. García-Centeno et al., "Expression and potential role of apolipoprotein D on the death-survival balance of human colorectal cancer cells under oxidative stress conditions," International Journal of Colorectal Disease, vol. 28, no. 6, pp. 751-766, 2013.

[42] C. Lebrero-Fernández, U. Wenzel, P. Akeus et al., “Altered expression of butyrophilin (BTN) and BTN-like (BTNL) genes in intestinal inflammation and colon cancer," Immunity, Inflammation and Disease, vol. 4, no. 2, pp. 191-200, 2016.

[43] G. Blandino and S. Di Agostino, "New therapeutic strategies to treat human cancers expressing mutant p 53 proteins," Journal of Experimental Clinical Cancer Research, vol. 37, no. 1, p. 30, 2018.

[44] E. Garcia, K. Simões, A. Wakamatsu et al., "Lymphatic vessel density and VEGF-C expression are significantly different among benign and malignant thyroid lesions," Endocrine Pathology, vol. 21, no. 2, pp. 101-107, 2010.

[45] V. de Araujo-Filho, V. Alves, I. de Castro et al., "Vascular endothelial growth factor expression in invasive papillary thyroid carcinoma," Thyroid, vol. 19, no. 11, pp. 1233-1237, 2009.
[46] Z. Karaca, F. Tanriverdi, K. Unluhizarci et al., "VEGFR1 expression is related to lymph node metastasis and serum VEGF may be a marker of progression in the follow-up of patients with differentiated thyroid carcinoma," European Journal of Endocrinology, vol. 164, no. 2, pp. 277-284, 2011.

[47] K. Pathak, A. Mazurat, P. Lambert, T. Klonisch, and R. Nason, "Prognostic nomograms to predict oncological outcome of thyroid cancers," The Journal of Clinical Endocrinology Metabolism, vol. 98, no. 12, pp. 4768-4775, 2013.

[48] Y. Ding, Z. Mao, J. Ruan et al., "Nomogram-based new recurrence predicting system in early-stage papillary thyroid cancer," International Journal of Endocrinology, vol. 2019, Article ID 1029092, 7 pages, 2019. 